\title{
Controllability of Passive Single-Input Single-Output Systems
}

\author{
Timothy H. Hughes ${ }^{1}$
}

\begin{abstract}
We provide a complete set of necessary and sufficient conditions for both conservative (lossless) and passive single-input single-output systems (Theorems 5 and 7, respectively). These conditions generalise the famous lossless positivereal lemma and positive-real lemma, respectively, by extending them to include uncontrollable systems.
\end{abstract}

\section{INTRODUCTION}

Passivity is a fundamental concept pertaining to physical systems: there is a limit to the net amount of energy which can be extracted from a passive system. A related concept is the notion of a conservative (lossless) system, which is a passive system for which there is a zero net energy transfer whenever the initial and final conditions of the system are identical. The central role of passive and conservative systems in systems and control is exemplified by the celebrated positive-real lemma, sometimes called the KalmanYakubovich-Popov (KYP) lemma, and its counterpart: the lossless positive-real lemma. These lemmas both demonstrate the equivalence of a time domain, a frequency domain, and an integral condition, for linear time-invariant systems. The integral conditions in the positive-real and lossless positivereal lemmas reflect the notions of passive and conservative systems, respectively. However, these lemmas are typically stated for controllable systems. As emphasised in [1], there is no explicit link between the concepts of passivity and controllability. Indeed, it is straightforward to construct examples of passive electric circuits whose driving-point behaviors are uncontrollable in a behavioral sense [1]-[4]. Moreover, the behavior of these electric circuits can often be described using a state-space realization in which the inductor currents and capacitor voltages are states, yet this state-space realization is neither controllable nor observable. There have been several valuable contributions towards a relaxation of the assumptions of the positive-real and lossless positive-real lemmas, but no complete set of necessary and sufficient conditions for a (not necessarily controllable) system to be passive is apparent. The purpose of this paper is to provide such necessary and sufficient conditions for the case of single-input single-output (SISO) systems. In addition to the preceding motivation, the results in this paper answer the question: what behaviors are realizable as the driving-point behavior of a one-port circuit comprising passive resistors, capacitors, inductors, transformers, and gyrators (c.f. the first open problem in [1]).

*This work was completed while the author was a Henslow Research Fellow, supported by the Cambridge Philosophical Society, www.cambridgephilosophicalsociety.org

${ }^{1}$ Timothy H. Hughes is with the Department of Engineering, University of Cambridge, Cambridge, CB2 1PZ, UK thh22 @ cam.ac.uk
The main original contributions of this paper are Theorems 5 and 7, both of which are concerned with SISO systems. Theorem 5 shows that controllability, which is stated as an assumption in the lossless positive-real lemma, is in fact a necessary condition for a system to be lossless. Theorem 7 removes the assumptions of controllability and observability from the positive-real lemma. For each of these two theorems, we demonstrate the equivalence of (i) a time-domain condition expressed as a linear matrix inequality (LMI) in terms of a state-space realization for the behavior; (ii) a condition relating to the differential equations determining the behavior, which generalises the known frequency-domain conditions; and (iii) an integral condition which captures the notion of a passive/conservative behavior.

Prior to stating and proving these two main theorems in Sections V and VI, we outline some necessary background material on behaviors (Section II), passivity (Section III), and bilinear differential forms (Section IV).

Throughout this paper, we adopt the following notation. $\mathbb{R}$ and $\mathbb{C}$ denote the real and complex numbers. $\mathbb{C}_{-}, \overline{\mathbb{C}}_{-}$, $\mathbb{C}_{+}, \overline{\mathbb{C}}_{+}$denote the open left half, closed left half, open right half, and closed right half plane, respectively. $\mathbb{R}[s]$ and $\mathbb{R}(s)$ denote the polynomials and rational functions in the indeterminate $s$ with real coefficients. For $z \in \mathbb{C}, \Re(z)$ denotes its real part, $\Im(z)$ its imaginary part, and $\bar{z}$ its conjugate. $\mathbb{F}^{m}$ and $\mathbb{F}^{m \times n}$ denote vectors and matrices of the indicated dimensions and with entries from $\mathbb{F}$ ( $\mathbb{F}$ can be any one of $\mathbb{R}, \mathbb{C}, \mathbb{R}[s]$ or $\mathbb{R}(s)$ ); • will be used in place of the dimensions when these are immaterial. For $M \in \mathbb{C}^{\bullet} \bullet \bullet$, $M^{*}$ denotes its Hermitian transpose, and $M>0$ and $M \geq 0$ indicate that $M$ is positive and non-negative definite, respectively. We let col $\left(\begin{array}{lll}M_{1} & \cdots & M_{n}\end{array}\right)=\left[\begin{array}{lll}M_{1}^{T} & \cdots & M_{n}^{T}\end{array}\right]^{T}$, and $\operatorname{diag}\left(M_{1} \cdots M_{n}\right)$ denotes the block diagonal matrix with $M_{1}, \ldots, M_{n}$ arranged in this order on the main diagonal. $\mathcal{L}_{1}^{\text {loc }}\left(\mathbb{R}, \mathbb{R}^{\bullet}\right)$ and $\mathcal{C}^{\infty}\left(\mathbb{R}, \mathbb{R}^{\bullet}\right)$ denote the (vectorvalued) locally integrable and infinitely differentiable functions [5, Definitions 2.3.3, 2.3.4]. A (vector-valued) $\mathrm{w}$ is called absolutely continuous (denoted $\mathbf{w} \in A C\left(\mathbb{R}, \mathbb{R}^{\bullet}\right)$ ) if (i) $\mathbf{w}$ is continuous; (ii) $\mathbf{w}$ is differentiable for all $\mathbb{R}$ with the possible exception of a set of measure zero; and (iii) for any given $t_{0} \in \mathbb{R}$, the derivative $\frac{d \mathbf{w}}{d t}$ satisfies $\mathbf{w}\left(t_{1}\right)=$ $\mathbf{w}\left(t_{0}\right)+\int_{t_{0}}^{t_{1}} \frac{d \mathbf{w}}{d t}(t) d t$ for all $t_{1} \geq t_{0}$ (the fundamental theorem of calculus).

\section{BEHAVIORS}

The behavioral approach [5], [6] provides a natural framework for the study of linear time-invariant differential behaviors. In this section, we outline those aspects of behavioral 
theory of relevance to this paper: controllability, stabilizability, observability, and input-state-output representations.

We will consider systems (behaviors) $\mathcal{B}$ defined as the set of locally integrable weak solutions (see [5, Section 2.3.2]) to a system of linear time-invariant differential equations, i.e.

$$
\mathcal{B}:=\left\{w \in \mathcal{L}_{1}^{\text {loc }}\left(\mathbb{R}, \mathbb{R}^{\bullet}\right) \mid R\left(\frac{d}{d t}\right) w=0, R \in \mathbb{R}^{\bullet \times} \bullet[s]\right\} .
$$

At times, we will also consider the subset of $\mathcal{B}$ comprised of the infinitely differentiable solutions to $R\left(\frac{d}{d t}\right) w=0$, which we denote by $\mathcal{B} \cap \mathcal{C}^{\infty}\left(\mathbb{R}, \mathbb{R}^{\bullet}\right)$. We note that any element in $\mathcal{B} \cap \mathcal{C}^{\infty}\left(\mathbb{R}, \mathbb{R}^{\bullet}\right)$ is also a solution to $R\left(\frac{d}{d t}\right)$ in the usual (strong) sense [5, Theorem 2.3.11].

We call the behavior $\mathcal{B}$ controllable if for any two trajectories $w_{1}, w_{2} \in \mathcal{B}$, and any $t_{1} \in \mathbb{R}$, there exists a $t_{2} \geq t_{1}$ and a $w \in \mathcal{B}$ which satisfies $w(t)=w_{1}(t)$ for all $t \leq t_{1}$ and $w(t)=w_{2}(t)$ for all $t \geq t_{2}$ [5, Definition 5.2.2]. $\mathcal{B}$ is called stabilizable if for every $w_{1} \in \mathcal{B}$, there exists a $w \in \mathcal{B}$ which satisfies $w(t)=w_{1}(t)$ for all $t \leq t_{1}$ and $\lim _{t \rightarrow \infty} w(t)=0$ [5, Definition 5.2.29]. The behavior $\mathcal{B}$ in (1) is controllable (resp., stabilizable) if and only if the rank of $R(\xi)$ is the same for all $\xi \in \mathbb{C}$ (resp., $\xi \in \overline{\mathbb{C}}_{+}$) [5, Thms 5.2.10 and 5.2.30].

In this paper, our focus is predominantly on single-input single-output (SISO) systems. These take the general form

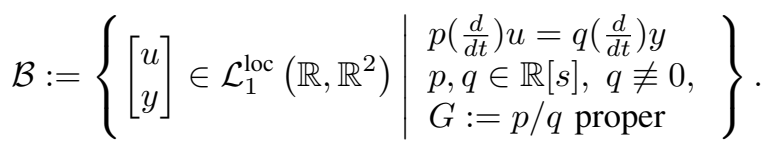

We will refer to any system which cannot be represented in the above form as a multi-input multi-output (MIMO) system. Evidently, the behavior $\mathcal{B}$ in (2) is controllable (resp., stabilizable) if and only if $p$ and $q$ are coprime (resp., $p$ and $q$ have no common roots in $\overline{\mathbb{C}}_{+}$).

Any behavior $\mathcal{B}$ as in (1) has an input-state-output representation (a realization). Specifically, the system's variables may be partitioned into inputs $u$ and outputs $y$ such that

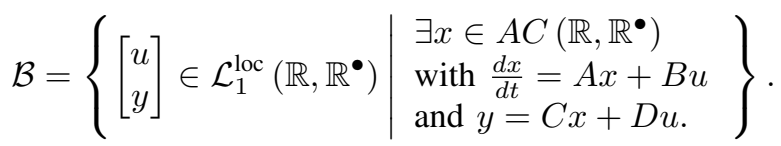

Here, every input $u \in \mathcal{L}_{1}^{\text {loc }}\left(\mathbb{R}, \mathbb{R}^{\bullet}\right)$ and every initial state $x(0) \in \mathbb{R}^{\bullet}$ determine a unique solution to (3). Specifically, $x$ is given by the variation of the constants formula [5, Corollary 4.5.5]:

$$
x(t)=e^{A t} x(0)+\int_{0}^{t} e^{A(t-\tau)} B u(\tau) d \tau \text { for all } t \in \mathbb{R},
$$

whereupon the 'output' $y$ is determined from $y=C x+D u$. We say that the pair $(A, B)$ is controllable (resp., stabilizable) whenever $\left[\begin{array}{ll}\lambda I-A & B\end{array}\right]$ has full row rank for all $\lambda \in \mathbb{C}$ (resp., $\lambda \in \overline{\mathbb{C}}_{+}$). Clearly, $(A, B)$ is controllable (resp., stabilizable) if and only if the behavior $\mathcal{B}_{s}:=\left\{\operatorname{col}\left(\begin{array}{ll}x & u\end{array}\right) \in\right.$ $\left.\mathcal{L}_{1}^{\text {loc }}\left(\mathbb{R}, \mathbb{R}^{\bullet}\right) \mid \frac{d x}{d t}=A x+B u\right\}$ is controllable. The realization (3) is called observable if, whenever col $\left(\begin{array}{lll}x_{1} & u & y\end{array}\right)$ and $\operatorname{col}\left(\begin{array}{lll}x_{2} & u & y\end{array}\right)$ are two solutions to (3), then $x_{1} \equiv x_{2}$. From [5, Section 5.3], the realization (3) is observable whenever $\operatorname{col}(\lambda I-A \quad C)$ has full column rank for all $\lambda \in \mathbb{C}$, in which case we also refer to the pair $(C, A)$ as being observable. For SISO systems (2), an observable realization is given by the observer canonical form [5, Theorem 6.4.2], and observable realizations also exist for any MIMO system [7], [8]. If the realization (3) of $\mathcal{B}$ is not observable, then there exists a non-singular matrix $T$ such that $\hat{A}, \hat{B}$ and $\hat{C}$, partitioned compatibly, take the form

$$
\hat{A}=\left[\begin{array}{cc}
\hat{A}_{11} & \hat{A}_{12} \\
0 & \hat{A}_{22}
\end{array}\right], \hat{B}=\left[\begin{array}{l}
\hat{B}_{1} \\
\hat{B}_{2}
\end{array}\right] \text {, and } \hat{C}=\left[\begin{array}{ll}
0 & \hat{C}_{2}
\end{array}\right],
$$

with $\hat{A}=T A T^{-1}, \hat{B}=T B$, and $\hat{C}=C T^{-1}$,

and where $\left(\hat{C}_{2}, \hat{A}_{22}\right)$ is observable [5, Corollary 5.3.14]. Then, using the variation of the constants formula (4), it is easily shown that $\mathcal{B}$ also has an observable realization:

$$
\mathcal{B}=\left\{\left[\begin{array}{l}
u \\
y
\end{array}\right] \in \mathcal{L}_{1}^{\text {loc }}\left(\mathbb{R}, \mathbb{R}^{\bullet}\right) \begin{array}{l}
\exists \hat{x}_{2} \in A C\left(\mathbb{R}, \mathbb{R}^{\bullet}\right): \\
\frac{d \hat{x}_{2}}{d t}=\hat{A}_{22} \hat{x}_{2}+\hat{B}_{2} u, \\
y=\hat{C}_{2} \hat{x}_{2}+D u .
\end{array}\right\} .
$$

\section{PASSIVE AND LOSSLESS SYSTEMS}

The physically motivated concepts of passive and conservative (lossless) systems play a key role in systems and control through the famous positive-real lemma and lossless positive-real lemma, respectively.

Theorem 1 (Positive-real lemma): Let $\mathcal{B}$ be as in (3) with $(A, B)$ controllable and $(C, A)$ observable and with $u, y \in$ $\mathcal{L}_{1}^{\text {loc }}\left(\mathbb{R}, \mathbb{R}^{m}\right)$. Then the following are equivalent:

1) $\int_{-\infty}^{t_{1}} u^{T}(t) y(t) d t \geq 0$ for all $t_{1} \in \mathbb{R}$ and col $\left(\begin{array}{ll}u & y\end{array}\right) \in \mathcal{B}$ which have bounded support on the left.

2) There exists a function $S$ which satisfies

$$
\int_{t_{0}}^{t_{1}} u^{T}(t) y(t) d t \geq S\left(x\left(t_{1}\right)\right)-S\left(x\left(t_{0}\right)\right),
$$

for all $t_{1} \geq t_{0} \in \mathbb{R}$ and $\operatorname{col}\left(\begin{array}{lll}x & u & y\end{array}\right)$ satisfying (3), and $S\left(x_{0}\right) \geq 0$ for all $x_{0} \in \mathbb{R}^{\bullet}$..

3) $G(s):=D+C(s I-A)^{-1} B$ satisfies $G(\lambda)^{*}+G(\lambda) \geq 0$ for all $\lambda \in \mathbb{C}_{+}$.

4) There exists a $P>0$ such that

$$
Q:=\left[\begin{array}{cc}
-A^{T} P-P A & C^{T}-P B \\
C-B^{T} P & D+D^{T}
\end{array}\right] \geq 0,
$$

and $S(x):=x^{T} P x$ is a non-negative storage function. Proof: See [9, Sections 3 to 5] and [10].

We say that $G \in \mathbb{R}^{m \times m}(s)$ is positive-real (PR) whenever $G$ has the properties outlined in condition 3 of Theorem 1 (we note that this condition implies that $G$ is analytic in $\mathbb{C}_{+}$, since if $G$ has a pole at some $\lambda \in \mathbb{C}_{+}$, then it may be shown that, for any $\epsilon>0$, there exists $\mathbf{z} \in \mathbb{C}^{m}$ and an $\eta \in \mathbb{C}$ with $|\eta|<\epsilon$ such that $\left.\mathbf{z}^{*}\left(G(\lambda+\eta)^{*}+G(\lambda+\eta)\right) \mathbf{z}<0\right)$. We will call the function $S$ defined in condition 2 of the above theorem a non-negative storage function. Furthermore, with $P, Q$ as defined in condition 4 , it may be verified that $S(x):=x^{T} P x$ is a non-negative storage function, which we will call a quadratic state storage function.

Theorem 2 (Lossless positive-real lemma): Let $\mathcal{B}$ be as in (3) with $(A, B)$ controllable and $(C, A)$ observable and with $u, y \in \mathcal{L}_{1}^{\text {loc }}\left(\mathbb{R}, \mathbb{R}^{m}\right)$. Then the following are equivalent: 
1) There exists a non-negative storage function $S$ with $\int_{t_{0}}^{t_{1}} u^{T}(t) y(t) d t=S\left(x\left(t_{1}\right)\right)-S\left(x\left(t_{0}\right)\right)$ for all $t_{1} \geq$ $t_{0} \in \mathbb{R}$ and col $\left(\begin{array}{lll}x & u & y\end{array}\right)$ satisfying (3).

2) $G(s):=D+C(s I-A)^{-1} B$ is PR and satisfies $G(s)+$ $G^{T}(-s) \equiv 0$.

3) There exists a $P>0$ such that $Q$ in (8) satisfies $Q=0$. Proof: See [9, Section 7] and [10].

We say that $G \in \mathbb{R}^{m \times m}(s)$ is lossless positive-real (LPR) whenever $G$ has the properties outlined in condition 2 of Theorem 2.

Condition 1 of Theorem 1 is reminiscent of the concept of passivity: the net energy which can be extracted from the system, when starting at rest, is bounded above by zero. In fact, this is adopted as the definition of passivity by some authors [10], [11]. Conversely, in this paper, we adopt the definition of a passive behavior proposed in [3], [4]. Below, we state this definition for SISO systems (which are the main concern of this paper).

Definition 3 (Passive behavior): $\mathcal{B}$ in (2) is passive if, for any given $\operatorname{col}\left(\begin{array}{ll}u & y\end{array}\right) \in \mathcal{B}$ and $t_{0} \in \mathbb{R}$, there exists a

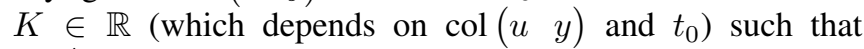
$-\int_{t_{0}}^{t_{1}} u(t) y(t) d t \leq K$ for all $t_{1} \geq t_{0}$.

It is our contention that this definition reflects the fundamental property associated with passivity: there is a limit to the net amount of energy which can be extracted from the system (irrespective of the initial condition). We then define a (SISO) lossless behavior as follows.

Definition 4 (Lossless behavior): $\mathcal{B}$ in (2) is lossless if it is passive (see Definition 3), and if $\int_{t_{0}}^{t_{0}+n T} u(t) y(t) d t=$ 0 for every single integer $n$ whenever $\operatorname{col}\left(\begin{array}{ll}u & y\end{array}\right) \in \mathcal{B}$ is periodic with period $T$.

The above definition captures the fundamental property of a conservative (lossless) system: there is a zero net energy transfer whenever the initial and final conditions of the system are identical. For linear systems, this condition may be replaced with $\int_{-\infty}^{\infty} u^{T}(t) y(t) d t=0$ whenever $\operatorname{col}\left(\begin{array}{ll}u & y\end{array}\right) \in$ $\mathcal{B}$ has compact support; i.e. there is a zero net energy transfer whenever the system starts and ends at rest.

Note that there are behaviors which satisfy condition 1 of Theorem 1 but are not passive in accordance with Definition 3. For example, for $\mathcal{B}:=\left\{\operatorname{col}\left(\begin{array}{ll}u & y\end{array}\right) \in \mathcal{L}_{1}^{\text {loc }}\left(\mathbb{R}, \mathbb{R}^{2}\right) \mid \frac{d y}{d t}=\right.$ $\left.\frac{d u}{d t}\right\}$, then any element in $\mathcal{B}$ takes the form $\operatorname{col}\left(\begin{array}{ll}u & u+k)\end{array}\right.$ for some $k \in \mathbb{R}$, whence those elements with bounded support on the left have $k=0$ and $\int_{-\infty}^{t_{1}} u(t) y(t) d t=$ $\int_{-\infty}^{t_{1}} u^{2}(t) d t \geq 0$. However, with $u(t)=-1$ and $y(t)=1$

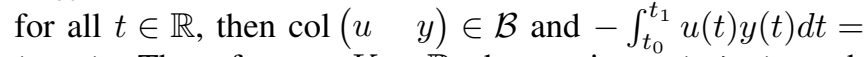
$t_{1}-t_{0}$. Thus, for any $K \in \mathbb{R}$, there exists a $t_{1} \geq t_{0}$ such that $-\int_{t_{0}}^{t_{1}} u(t) y(t) d t>K$, and so $\mathcal{B}$ is not passive. However, for controllable systems, Definition 3 is in fact equivalent to condition 1 of Theorem 1. This is discussed in [3] but no proof or reference is provided; for SISO systems this follows from the proof of Theorem 5 of this paper.

We remark that observability is of little consequence in Theorem 1. Indeed, it is shown in [12] that conditions 1, 2, and 4 of Theorem 1 are equivalent irrespective of whether $(C, A)$ is observable providing we relax the condition on $P$ to $P \geq 0$. Moreover, it is shown that any one of these three conditions imply condition 3 irrespective of observability, but no proof was provided of the converse implication [12], [13].

The assumption of controllability in Theorem 1 is much less easy to dispense of. For example, consider again the behavior $\mathcal{B}:=\left\{\operatorname{col}\left(\begin{array}{ll}u & y\end{array}\right) \in \mathcal{L}_{1}^{\text {loc }}\left(\mathbb{R}, \mathbb{R}^{2}\right) \mid \frac{d y}{d t}=\frac{d u}{d t}\right\}$, and let $\mathcal{B}$ have an observable realization as in (3). From before, $\mathcal{B}$ satisfies condition 1 of Theorem 1 , yet $\mathcal{B}$ is not passive in accordance with Definition 3. Moreover, it follows as a consequence of Theorem 7 of this paper that conditions 2 and 4 do not hold for this behavior. In other words, conditions 1 to 4 are not equivalent when considering uncontrollable behaviors. The purpose of this paper is to resolve these issues in the context of SISO systems. In particular, Theorem 5 extends the lossless positive-real lemma (Theorem 2), and Theorem 7 extends the positive-real lemma (Theorem 1), to cover general (not necessarily controllable) SISO systems.

There have been several contributions in the literature which have sought to relax the assumption of controllability in the positive-real lemma. On the one hand, several papers have sought to replace this with a weaker assumption (see [14]-[16] and the references therein). These assumptions are specified a-priori rather than being motivated by passivity itself. On the other hand, [17] showed the equivalence of: 3a) $G(s)$ is analytic in $\overline{\mathbb{C}}_{+}$and satisfies $G(\xi)^{*}+G(\xi)>0$ for all $\xi \in \overline{\mathbb{C}}_{+} \cup \infty$; and 4 a) there exists a $P>0$ such that $Q$ in (8) satisfies $Q>0$. There is still an important class of passive systems which are not considered by these contributions, for example none of these papers consider lossless systems. Moreover, to connect the results with physical systems, it is necessary to establish the equivalence of these conditions with an integral condition capturing the concept of passivity. In this paper we show that, in the SISO case (2), the assumption of controllability in the positive-real lemma may be removed providing we augment the frequency domain condition (condition 3 in Theorem 1) with a condition on the location of the common divisors of $p$ and $q$ (this is condition 3 of Theorem 7).

We note that Theorem 1 has a companion theorem which considers the case in which the storage function can take all real values. The systems covered by this latter theorem are not necessarily passive (they are sometimes called pseudopassive systems, and are a special case of cyclo-dissipative systems [3]). Nevertheless, this theorem is also referred to as the positive-real lemma or KYP lemma by some authors (e.g. [18], [19]). There have have also been several notable contributions concerned with relaxing the assumption of controllability for pseudo-passive/cyclo-dissipative systems (see [18], [19] and the references therein). The papers [1], [20], [21] adopt a behavioral approach to this problem using the framework of quadratic differential forms. However, as is the case for passive systems, no theorem is apparent which does not require any extraneous assumptions.

\section{BILINEAR DIFFERENTIAL FORMS}

The framework of bilinear differential forms (BDFs) was proposed in [22] for studying dissipativity. Here, we intro- 
duce relevant terminology for BDFs which will be used in subsequent sections.

A bilinear differential form is a mapping from $\mathcal{C}_{\infty}\left(\mathbb{R}, \mathbb{R}^{\bullet}\right) \times \mathcal{C}_{\infty}\left(\mathbb{R}, \mathbb{R}^{\bullet}\right)$ to $\mathcal{C}_{\infty}(\mathbb{R}, \mathbb{R})$ of the form:

$$
L_{\Phi}(w, x):=\sum_{i=1}^{M} \sum_{j=1}^{N}\left(\frac{d^{i-1} w}{d t^{i-1}}\right)^{T} \Phi_{i j}\left(\frac{d^{j-1} x}{d t^{j-1}}\right),
$$

for some integers $M, N$ and real-valued matrices $\Phi_{i j}$. Such a BDF is naturally associated with the two variable polynomial matrix $\Phi \in \mathbb{R}^{\bullet \bullet} \bullet[\xi, \eta]$ defined as $\Phi(\xi, \eta):=$ $\sum_{i=1}^{M} \sum_{j=1}^{N} \Phi_{i j} \xi^{i-1} \eta^{j-1}$. We then note that the product rule of differentiation yields:

$\frac{d}{d t} L_{\Phi}(w, x)=L_{\Psi}(w, x)$, for $\Psi(\xi, \eta):=(\xi+\eta) \Phi(\xi, \eta)$.

The above relationship can be used to derive a special BDF which is associated with a generalised integration by parts formula. We introduce this in the context of scalar differential equations below; a generalisation to systems of differential equations is discussed in [23]. To any given $r \in \mathbb{R}[s]$ we associate the $\operatorname{BDF} L_{\Phi_{r}}$, where $\Phi_{r} \in \mathbb{R}[\xi, \eta]$ satisfies

$$
\Phi_{r}(\xi, \eta)=(r(\xi)-r(-\eta)) /(\xi+\eta) .
$$

It follows from the factor theorem that $\Phi_{r}$ is a two variable polynomial matrix, since $r(\xi)-r(-\eta)=0$ whenever $\xi+$ $\eta=0$. From (9), we obtain $\left(r\left(\frac{d}{d t}\right) w\right) x-w\left(r\left(-\frac{d}{d t}\right) x\right)=$ $\frac{d}{d t} L_{\Phi_{r}}(w, x)$. Thus, with the notation $\left[L_{\Phi_{r}}(w, x)(t)\right]_{t_{0}}^{t_{1}}:=$ $L_{\Phi_{r}}(w, x)\left(t_{1}\right)-L_{\Phi_{r}}(w, x)\left(t_{0}\right)$, we obtain

$$
\begin{aligned}
& \int_{t_{0}}^{t_{1}}\left(r\left(\frac{d}{d t}\right) w\right)(t) x(t) d t \\
& =\int_{t_{0}}^{t_{1}} w(t)\left(r\left(-\frac{d}{d t}\right) x\right)(t) d t+\left[L_{\Phi_{r}}(w, x)(t)\right]_{t_{0}}^{t_{1}}
\end{aligned}
$$

Note that in the case $r\left(\frac{d}{d t}\right)=\frac{d}{d t}$, we obtain $\Phi_{r} \equiv 1$, and (10) becomes the formula for integration by parts.

\section{ALL LOSSLESS BEHAVIORS ARE CONTROLLABLE}

In this section, we show that any lossless SISO behavior is necessarily controllable, i.e. $p$ and $q$ are coprime in (2). This leads to Theorem 5 below, which extends the lossless positive-real lemma (Theorem 2) to cover general (not necessarily controllable) SISO systems.

Theorem 5: For $\mathcal{B}$ as in (2), the following are equivalent:

1) $\mathcal{B}$ is lossless (see Definition 4 ).

2) $\mathcal{B}$ has an observable realization as in (3), and for any such realization there exists a function $S$ such that $\int_{t_{0}}^{t_{1}} u(t) y(t) d t=S\left(x\left(t_{1}\right)\right)-S\left(x\left(t_{0}\right)\right)$ for all $t_{1} \geq$ $t_{0} \in \mathbb{R}$ and col $\left(\begin{array}{lll}x & u & y\end{array}\right)$ satisfying (3), and where $S\left(x_{0}\right) \geq 0$ for all $x_{0} \in \mathbb{R}^{\bullet}$.

3) $G$ is LPR and $p$ and $q$ are coprime.

4) $\mathcal{B}$ has an observable realization as in (3), and for any such realization there exists a $P>0$ such that $Q$ in (8) satisfies $Q=0$.

Our proof will use the following theorem, which was proved for MIMO systems in [4]:
Theorem 6: Let $\mathcal{B}$ in (2) be passive. Then $\mathcal{B}$ is stabilizable. In other words, $p$ and $q$ have no common roots in $\overline{\mathbb{C}}_{+}$.

Proof: See [4, Theorem 6].

Proof: [Theorem 5] We will show that $3 \Longrightarrow 4 \Longrightarrow$ $2 \Longrightarrow 1 \Longrightarrow 3$. Note initially that $3 \Longrightarrow 4$ by Theorem 2 as any observable realization of a controllable behavior necessarily has $(A, B)$ controllable (this follows from the proof of Theorem 5.2 in [8]). To see that $4 \Longrightarrow 2$ we note, since $x$ is absolutely continuous, that

$$
\begin{aligned}
\int_{t_{0}}^{t_{1}} u(t) y(t) d t=\frac{1}{2} \int_{t_{0}}^{t_{1}}\left[\begin{array}{l}
x(t) \\
u(t)
\end{array}\right]^{T} Q\left[\begin{array}{l}
x(t) \\
u(t)
\end{array}\right] d t \\
+\frac{1}{2}\left[x(t)^{T} P x(t)\right]_{t_{0}}^{t_{1}}
\end{aligned}
$$

It follows that $\left\{S \mid S\left(x_{0}\right)=x_{0}^{T} P x_{0}\right.$ for all $\left.x_{0} \in \mathbb{R}^{\bullet}\right\}$ has the properties outlined in condition 2. Also, from (11),

$$
-\int_{t_{0}}^{t_{1}} u(t) y(t) d t \leq \frac{1}{2}\left(x\left(t_{0}\right)^{T} P x\left(t_{0}\right)\right)
$$

for all $t_{1} \geq t_{0}$, which implies that $\mathcal{B}$ is passive. Now, let $\operatorname{col}\left(u_{1} y_{1}\right) \in \mathcal{B}$ be periodic with period $T$. Then $\operatorname{col}\left(\begin{array}{ll}u_{2} & y_{2}\end{array}\right) \in \mathcal{B}$ where $\operatorname{col}\left(\begin{array}{ll}u_{2} & y_{2}\end{array}\right)(t)=\operatorname{col}\left(\begin{array}{ll}u_{1} & y_{1}\end{array}\right)(t+$ $n T)=\operatorname{col}\left(\begin{array}{ll}u_{1} & y_{1}\end{array}\right)(t)$ for all $t \in \mathbb{R}$ and for any given integer $n$. Thus, whenever the realization (3) is observable, we have $x(t+n T)=x(t)$ for all $t \in \mathbb{R}$ and for any given integer $n$ (see Section II), whence (11) implies that $\int_{t_{0}}^{t_{0}+n T} u(t) y(t) d t=0$. We thus find that $2 \Longrightarrow 1$.

It remains to show that $1 \Longrightarrow 3$. Accordingly, we will show the following:

(i) If $\mathcal{B}$ is passive, then $G$ is PR.

(ii) If $\mathcal{B}$ is lossless, then $G$ is LPR.

(iii) If $\mathcal{B}$ is passive and $G$ is $\mathrm{LPR}$, then $\mathcal{B}$ is controllable.

To show (i), we consider an arbitrary $\lambda \in \mathbb{C}_{+}$, and we note that $\operatorname{col}\left(\begin{array}{ll}\hat{u} & \hat{y}\end{array}\right) \in \mathcal{B}$ where $\operatorname{col}\left(\begin{array}{ll}\hat{u} & \hat{y}\end{array}\right)(t)=$ $\Re\left(\operatorname{col}(q(\lambda) p(\lambda)) e^{\lambda t}\right)$ for all $t \in \mathbb{R}$. Thus, with the notation $\Phi(\xi, \eta):=(1 / 2)(p(\xi) q(\eta)+q(\xi) p(\eta))$, we find that

$$
\begin{aligned}
\int_{t_{0}}^{t_{1}} \hat{u}(t) \hat{y}(t) d t & =\frac{1}{2} \int_{t_{0}}^{t_{1}} \Re\left(\Phi(\lambda, \lambda) e^{2 \lambda t}\right)+\Phi(\lambda, \bar{\lambda}) e^{(\lambda+\bar{\lambda}) t} d t \\
& =\frac{1}{2}\left[\Re\left(\frac{\Phi(\lambda, \lambda) e^{2 \lambda t}}{2 \lambda}\right)+\frac{\Phi(\lambda, \bar{\lambda}) e^{(\lambda+\bar{\lambda}) t}}{\lambda+\bar{\lambda}}\right]_{t_{0}}^{t_{1}} .
\end{aligned}
$$

Now, suppose that $2 \Phi(\lambda, \bar{\lambda})=p(\lambda) q(\bar{\lambda})+q(\lambda) p(\bar{\lambda})<0$. Suppose initially that $|\Im(\lambda)|=\omega \neq 0$, let $T=\pi / \omega$, and let $t_{0}$ satisfy $2 \omega t_{0}+\arg (\Phi(\lambda, \lambda) / \lambda)=\pi / 2$ (so $\left.\Re\left(\Phi(\lambda, \lambda) e^{2 \lambda t_{0}} / 2 \lambda\right)=0\right)$. Then, for any $K \in \mathbb{R}$, there exists an integer $N$ such that

$$
-\int_{t_{0}}^{t_{0}+N T} \hat{u}(t) \hat{y}(t) d t=-\frac{1}{2}\left[\frac{\Phi(\lambda, \bar{\lambda}) e^{(\lambda+\bar{\lambda}) t}}{\lambda+\bar{\lambda}}\right]_{t_{0}}^{t_{0}+N T}>K,
$$

whence $\mathcal{B}$ is not passive. If, on the other hand, $\Im(\lambda)=0$, then $\lambda=\bar{\lambda}$, and again we find that for any $K \in \mathbb{R}$ there exists a $t_{1} \geq t_{0} \in \mathbb{R}$ such that $-\int_{t_{0}}^{t_{1}} \hat{u}(t) \hat{y}(t) d t>K$. It follows that if $\mathcal{B}$ is passive then $2 \Phi(\lambda, \bar{\lambda})=p(\lambda) q(\bar{\lambda})+q(\lambda) p(\bar{\lambda})$ is non-negative whenever $\lambda \in \mathbb{C}_{+}$. Since, in addition, $q \neq \equiv 0$ and $p, q \in \mathbb{R}[s]$, then $G(\lambda)^{*}+G(\lambda) \geq 0$ for all $\lambda \in \mathbb{C}_{+}$, whence $G$ is PR. 
To show (ii), we consider an arbitrary $0 \neq \omega \in \mathbb{R}$, we let $\operatorname{col}(\hat{u} \hat{y})(t)=\Re\left(\operatorname{col}(q(j \omega) p(j \omega)) e^{j \omega t}\right)$ for all $t \in \mathbb{R}$, and we note that $\operatorname{col}(\hat{u} \hat{y}) \in \mathcal{B}$ and $\operatorname{col}\left(\begin{array}{ll}\hat{u} & \hat{y}\end{array}\right)$ is periodic with period $T=2 \pi / \omega$. In this case, again with $\Phi(\xi, \eta):=(1 / 2)(p(\xi) q(\eta)+q(\xi) p(\eta))$, we find that $\int_{t_{0}}^{t_{0}+n T} \hat{u}(t) \hat{y}(t) d t=(1 / 2) \Phi(j \omega,-j \omega) n T$, whence we require $\Phi(j \omega,-j \omega)=0$ for all $0 \neq \omega \in \mathbb{R}$. This implies that $G(j \omega)^{*}+G(j \omega)=0$ all $0 \neq \omega \in \mathbb{R}$. Since, in addition $G \in \mathbb{R}(s)$, then this implies that $G(s)+G(-s) \equiv 0$, whence $G$ is LPR.

To show (iii), note initially from Theorem 6 that if $\mathcal{B}$ is not stabilisable then $\mathcal{B}$ is not passive. It remains to consider the case where $\mathcal{B}$ is stabilisable but not controllable, $\mathcal{B}$ is passive, and $G$ is LPR. Accordingly, let $g$ be the greatest common divisor of $p$ and $q$, and let $\hat{p}:=p / g$ and $\hat{q}:=q / g$, whence $\hat{p}, \hat{q} \in \mathbb{R}[s]$ and $\hat{p}$ and $\hat{q}$ are coprime. Then any col $\left(\begin{array}{ll}u & y\end{array}\right) \in$ $\mathcal{B}$ satisfies $g\left(\frac{d}{d t}\right)\left[-\hat{p}\left(\frac{d}{d t}\right) \quad \hat{q}\left(\frac{d}{d t}\right)\right] \operatorname{col}\left(\begin{array}{ll}u & y\end{array}\right)=0$. Since $\mathcal{B}$ is stabilisable but not controllable, then $g$ has a root $\lambda \in \mathbb{C}_{-}$. We then let $\operatorname{col}\left(\begin{array}{ll}u_{1} & y_{1}\end{array}\right)(t)=\Re\left(\operatorname{col}\left(\begin{array}{ll}\tilde{u} & \tilde{y}\end{array}\right) e^{\lambda t}\right)$ for all $t \in$ $\mathbb{R}$ and for some fixed but arbitrary $\tilde{u}, \tilde{y} \in \mathbb{C}$, and it follows that $\operatorname{col}\left(u_{1} y_{1}\right) \in \mathcal{B}$. Moreover, whenever $z \in \mathcal{C}^{\infty}(\mathbb{R}, \mathbb{R})$, then $g\left(\frac{d}{d t}\right)\left[-\hat{p}\left(\frac{d}{d t}\right) \quad \hat{q}\left(\frac{d}{d t}\right)\right] \operatorname{col}\left(\hat{q}\left(\frac{d}{d t}\right) \quad \hat{p}\left(\frac{d}{d t}\right)\right) z=0$, and so $\operatorname{col}\left(u_{2} y_{2}\right)=\operatorname{col}\left(\hat{q}\left(\frac{d}{d t}\right) \quad \hat{p}\left(\frac{d}{d t}\right)\right) z$ is also in $\mathcal{B}$. By linearity, $\operatorname{col}\left(\begin{array}{ll}u & y\end{array}\right):=\operatorname{col}\left(u_{1}+u_{2} y_{1}+y_{2}\right) \in \mathcal{B}$. Here,

$$
\begin{aligned}
\int_{t_{0}}^{t_{1}} u(t) y(t) d t & =J_{1}+J_{2}+J_{3}+J_{4}, \\
\text { where } J_{1} & :=\int_{t_{0}}^{t_{1}}\left(\hat{q}\left(\frac{d}{d t}\right) z\right)(t)\left(\hat{p}\left(\frac{d}{d t}\right) z\right)(t) d t, \\
J_{2} & :=\int_{t_{0}}^{t_{1}} \Re\left(\tilde{u} e^{\lambda t}\right)\left(\hat{p}\left(\frac{d}{d t}\right) z\right)(t) d t, \\
J_{3} & :=\int_{t_{0}}^{t_{1}}\left(\hat{q}\left(\frac{d}{d t}\right) z\right)(t) \Re\left(\tilde{y} e^{\lambda t}\right) d t, \\
\text { and } J_{4} & :=\int_{t_{0}}^{t_{1}} \Re\left(\tilde{u} e^{\lambda t}\right) \Re\left(\tilde{y} e^{\lambda t}\right) d t .
\end{aligned}
$$

Consider first $J_{1}$, and note that this clearly satisfies $J_{1}=$ $(1 / 2) \int_{t_{0}}^{t_{1}}\left(\hat{q}\left(\frac{d}{d t}\right) z\right)(t)\left(\hat{p}\left(\frac{d}{d t}\right) z\right)(t)+\left(\hat{p}\left(\frac{d}{d t}\right) z\right)(t)\left(\hat{q}\left(\frac{d}{d t}\right) z\right)(t) d t$ By initially setting $r=\hat{p}, w=z$, and $x=\hat{q}\left(\frac{d}{d t}\right) z$ in (10), and then setting $r=\hat{q}, w=z$, and $x=\hat{p}\left(\frac{d}{d t}\right) z$, we obtain $J_{1}=\frac{1}{2} \int_{t_{0}}^{t_{1}} z(t)\left(\left(\hat{p}\left(-\frac{d}{d t}\right) \hat{q}\left(\frac{d}{d t}\right)+\hat{q}\left(-\frac{d}{d t}\right) \hat{p}\left(\frac{d}{d t}\right)\right) z\right)(t)+$ $\frac{1}{2}\left[L_{\Phi_{\hat{p}}}\left(z, \hat{q}\left(\frac{d}{d t}\right) z\right)(t)+L_{\Phi_{\hat{q}}}\left(z, \hat{p}\left(\frac{d}{d t}\right) z\right)(t)\right]_{t_{0}}^{t_{1}}$. Since $q$ is non-zero and $p / q$ is LPR, then $\hat{q}$ is non-zero and $\hat{p} / \hat{q}$ is LPR, whence $\hat{p}(-s) \hat{q}(s)+\hat{q}(-s) \hat{p}(s) \equiv 0$, and

$$
J_{1}=\frac{1}{2}\left[L_{\Phi_{\hat{p}}}\left(z, \hat{q}\left(\frac{d}{d t}\right) z\right)(t)+L_{\Phi_{\hat{q}}}\left(z, \hat{p}\left(\frac{d}{d t}\right) z\right)(t)\right]_{t_{0}}^{t_{1}} .
$$

For $J_{2}$ we let $r=\hat{p}, w=z$, and $x=u_{1}$ (i.e. $x(t)=\Re\left(\tilde{u} e^{\lambda t}\right)$ for all $t \in \mathbb{R})$ in (10); and for $J_{3}$ we let $r=\hat{q}, w=z$, and $x=y_{1}$ (i.e. $x(t)=\Re\left(\tilde{y} e^{\lambda t}\right)$ for all $\left.t \in \mathbb{R}\right)$. Then

$$
\begin{aligned}
& J_{2}=\int_{t_{0}}^{t_{1}} z(t) \Re\left(\hat{p}(-\lambda) \tilde{u} e^{\lambda t}\right) d t+\left[L_{\Phi_{\hat{p}}}\left(z, u_{1}\right)(t)\right]_{t_{0}}^{t_{1}}, \text { and } \\
& J_{3}=\int_{t_{0}}^{t_{1}} z(t) \Re\left(\hat{q}(-\lambda) \tilde{y} e^{\lambda t}\right) d t+\left[L_{\Phi_{\hat{q}}}\left(z, y_{1}\right)(t)\right]_{t_{0}}^{t_{1}} . \quad \text { (19) }
\end{aligned}
$$

Now, let $z$ be chosen such that $\frac{d^{l} z}{d t^{l}}\left(t_{0}\right)=\frac{d^{l} z}{d t^{l}}\left(t_{1}\right)=0$ for $l=0,1,2, \ldots$. Then all of the BDFs in (18) and (19) are zero (i.e. $L_{\Phi_{\hat{p}}}\left(z, \hat{q}\left(\frac{d}{d t}\right) z\right)\left(t_{0}\right)=0$, and so forth). Furthermore, let $\tilde{u}, \tilde{y} \in \mathbb{C}$ satisfy $(\hat{p}(-\lambda) \tilde{u}+\hat{q}(-\lambda) \tilde{y})=1$ and $\Re\left(\tilde{u} e^{\lambda t}\right) \Re\left(\tilde{y} e^{\lambda t}\right)=0$ for all $t \in \mathbb{R}$ (if $\hat{p}(-\lambda) \neq 0$ then take $\tilde{u}=1 /(\hat{p}(-\lambda))$ and $\tilde{y}=0$, otherwise $\hat{q}(-\lambda) \neq 0$ since $\hat{p}$ and $\hat{q}$ are coprime, and we can take $\tilde{y}=1 /(\hat{q}(-\lambda))$ and $\tilde{u}=0$ ). It follows from (13)-(19) that $-\int_{t_{0}}^{t_{1}} u(t) y(t) d t=$ $-\int_{t_{0}}^{t_{1}} z(t) \Re\left(e^{\lambda t}\right) d t$.

To complete the proof of the present theorem, we show that, for any given $K \in \mathbb{R}$, there exists a $t_{1} \geq t_{0}$ and a $z \in \mathcal{C}^{\infty}(\mathbb{R}, \mathbb{R})$ with $\frac{d^{l} z}{d t^{l}}\left(t_{0}\right)=\frac{d^{l} z}{d t^{l}}\left(t_{1}\right)=0$ for $l=0,1,2, \ldots$ such that $-\int_{t_{0}}^{t_{1}} z(t) \Re\left(e^{\lambda t}\right) d t>K$. Let $f(t):=\Re\left(e^{\lambda t}\right)^{2}$ for all $t \in \mathbb{R}$, so $f(t)>0$ for all $t \in \mathbb{R}$ with the possible exception of a set of measure zero. Next, let $\phi(t)=e^{1 /\left(t^{2}-1\right)}$ for $-1<t<1$ with $\phi(t)=0$ otherwise (this is the bell-shaped function in [5, Fig. 2.5]). Further, for any given integer $k$, let $\eta_{k}(t)=f(t) \phi\left(t-1-t_{0}-2 k\right)$ for all $t \in \mathbb{R}$. It may be verified that $\eta_{k} \in \mathcal{C}^{\infty}(\mathbb{R}, \mathbb{R})$ and $\int_{t_{0}+2 k}^{t_{0}+2(k+1)} \eta_{k}(t) d t>0$. Now, let $N$ be a positive integer with $N>K$, and let $\psi(t)=-\sum_{k=0}^{N-1} \phi\left(t-1-t_{0}-2 k\right) /\left(\int_{t_{0}+2 k}^{t_{0}+2(k+1)} \eta_{k}(t) d t\right)$ for all $t \in \mathbb{R}$. It may be verified that $\psi \in \mathcal{C}^{\infty}(\mathbb{R}, \mathbb{R})$, that $\frac{d^{l} \psi}{d t^{l}}\left(t_{0}+2 k\right)=0$ for $k, l=0,1,2, \ldots$, and that $-\int_{t_{0}}^{t_{0}+2 N}(f \psi)(t) d t=N>K$. Accordingly, we let $z(t)=$ $\psi(t) \Re\left(e^{\lambda t}\right)$ for all $t \in \mathbb{R}$, and we find that $z \in \mathcal{C}^{\infty}(\mathbb{R}, \mathbb{R})$; $\frac{d^{l} z}{d t^{l}}\left(t_{0}\right)=\frac{d^{l} z}{d t^{l}}\left(t_{0}+2 N\right)=0(l=0,1,2, \ldots)$; and $-\int_{t_{0}}^{t_{0}+2 N} z(t) \Re\left(e^{\lambda t}\right) d t=-\int_{t_{0}}^{t_{0}+2 N}(f \psi)(t) d t>K$.

\section{NECESSARY AND SUFFICIENT CONDITIONS FOR A SISO SYSTEM TO BE PASSIVE}

In Theorems 5 and 6 , we showed that any given passive SISO behavior $\mathcal{B}$ as in (2) is necessarily stabilizable, and controllable whenever $\mathcal{B}$ is lossless. In this section, we show that these conditions, together with the condition that $G$ in (2) is $\mathrm{PR}$, are sufficient to ensure passivity. We thus obtain the following theorem, which extends the positive-real lemma to cover general (not necessarily controllable) SISO systems:

Theorem 7: For $\mathcal{B}$ as in (2), the following are equivalent:

1) $\mathcal{B}$ is passive (see Definition 3 ).

2) $\mathcal{B}$ has a realization as in (3), and for any such realization there exists a function $\mathrm{S}$ such that $\int_{t_{0}}^{t_{1}} u(t) y(t) d t \geq$ $S\left(x\left(t_{1}\right)\right)-S\left(x\left(t_{0}\right)\right)$ for all $t_{1} \geq t_{0} \in \mathbb{R}$ and $\operatorname{col}\left(\begin{array}{lll}x & u & y\end{array}\right)$ satisfying (3), and where $S\left(x_{0}\right) \geq 0$ for all $x_{0} \in \mathbb{R}^{\bullet}$.

3) $G$ is PR, and one of the following two conditions hold:

a) $G$ is not LPR and $\mathcal{B}$ is stabilizable. In other words, $G(s)+G(-s) \not \equiv 0$, and $p$ and $q$ have no common roots in $\overline{\mathbb{C}}_{+}$.

b) $G$ is LPR and $\mathcal{B}$ is controllable. In other words, $G(s)+G(-s) \equiv 0$ and $p$ and $q$ are coprime.

4) $\mathcal{B}$ has an observable realization as in (3), and for any such realization there exists a $P>0$ such that $Q$ in (8) satisfies $Q \geq 0$.

5) $\mathcal{B}$ has a (not necessarily observable) realization as in (3), and for any such realization there exists a $P \geq 0$ such that $Q$ in (8) satisfies $Q \geq 0$. 
We note that, for SISO passive behaviors, the above theorem demonstrates that the existence of a non-negative quadratic state storage function is equivalent to the existence of a non-negative quadratic observable state storage function. In contrast, we note there are systems which possess a (not necessarily non-negative) quadratic state storage function but do not possess a quadratic observable state storage function [1], [2], [18].

Finally, it is straightforward to show from results in [11] that condition 4 of Theorem 7 implies the existence of an electric circuit comprising resistors, inductors, capacitors, transformers, and gyrators whose driving-point behavior is equal to $\mathcal{B}$.

Proof: [Theorem 7] We will show that $5 \Longrightarrow 2 \Longrightarrow$ $1 \Longrightarrow 3 \Longrightarrow 4 \Longrightarrow 5$. That $5 \Longrightarrow 2$ follows from (11), which shows that $\left\{S \mid S\left(x_{0}\right)=x_{0}^{T} P x_{0}\right.$ for all $\left.x_{0} \in \mathbb{R}^{\bullet}\right\}$ has the properties outlined in condition 2 . To see that $2 \Longrightarrow 1$, we note from the first paragraph of the proof of Theorem 5 that (12) holds. That $1 \Longrightarrow 3$ follows from Theorems 5 and 6 . That $3 \mathrm{~b} \Longrightarrow 4$ was shown in Theorem 5 . It can be shown that whenever $\mathcal{B}$ is stabilisable then $\mathcal{B}$ has an observable realization as in (3) for which $(A, B)$ is stabilisable (the proof of this is similar to the proof of Theorem 5.2 in [8]). Since, in addition, $G$ is not LPR, then $G(s)+G(-s) \not \equiv 0$, and $3 \mathrm{a} \Longrightarrow 4$ by [20, Theorem 5].

It remains to show that $4 \Longrightarrow 5$. Accordingly, let (3) be a (not necessarily observable) realization of $\mathcal{B}$. Then, from Section II, there exists a non-singular matrix $T$ such that $\hat{A}, \hat{B}, \hat{C}$ in (6) take the form of (5), and where $\mathcal{B}$ has an observable realization as in (7). By condition 4 , this implies that there exists a $\hat{P}_{2}>0$ such that

$$
\hat{Q}_{2}:=\left[\begin{array}{cc}
-\hat{A}_{22}^{T} \hat{P}_{2}-\hat{P}_{2} \hat{A}_{22} & \hat{C}_{2}^{T}-\hat{P}_{2} \hat{B}_{2} \\
\hat{C}_{2}-\hat{B}_{2}^{T} \hat{P}_{2} & D+D^{T}
\end{array}\right] \geq 0 .
$$

Then, by direct calculation, $\hat{P}:=\operatorname{diag}\left(\begin{array}{ll}0 & \hat{P}_{2}\end{array}\right) \geq 0$ satisfies

$$
\hat{Q}:=\left[\begin{array}{cc}
-\hat{A}^{T} \hat{P}-\hat{P} \hat{A} & \hat{C}^{T}-\hat{P} \hat{B} \\
\hat{C}-\hat{B}^{T} \hat{P} & D+D^{T}
\end{array}\right]=\operatorname{diag}\left(\begin{array}{ll}
0 & \hat{Q}_{2}
\end{array}\right) \geq 0 .
$$

Finally, with $P:=T^{T} \hat{P} T \geq 0$, and with $S:=\operatorname{diag}\left(\begin{array}{ll}T & 1\end{array}\right)$, we find that $Q$ in (8) satisfies $Q=S^{T} \hat{Q} S \geq 0$.

\section{CONCLUSIONS AND EXTENSIONS}

We presented a theory of SISO conservative and passive linear systems which makes no controllability assumptions (Theorems 5 and 7). Notably, we showed that passivity (Definition 3) is equivalent to the existence of solutions to a particular linear matrix inequality. Moreover, if a SISO passive system is lossless then it is (behaviorally) controllable, and otherwise it is (behaviorally) stabilizable.

Theorem 5 extends to MIMO systems (this can be shown by making only minor adjustments to the proof in this paper). Theorem 7 can also be extended to MIMO systems with an amendment to condition 3. These extensions will be shown in the accompanying ECC presentation.

\section{REFERENCES}

[1] M. K. Çamlibel, J. C. Willems, and M. N. Belur, "On the dissipativity of uncontrollable systems," in Proceedings of the 42nd IEEE Conference on Decision and Control, Hawaii, Dec. 2003.

[2] J. C. Willems, "Hidden variables in dissipative systems," Proceedings of the 43rd IEEE Conference on Decision and Control, pp. 358 - 363, 2004.

[3] — , "Dissipative dynamical systems," European Journal on Control, vol. 13, pp. 134-151, 2007.

[4] T. H. Hughes and M. C. Smith, "Controllability of linear passive network behaviors," In press, Systems and Control Letters, doi:10.1016/j.sysconle.2015.09.011, 2015.

[5] J. W. Polderman and J. C. Willems, Introduction to Mathematical Systems Theory: A Behavioral Approach. New York : Springer-Verlag, 1998.

[6] J. C. Willems, "The behavioral approach to open and interconnected systems," Control Systems Magazine, vol. 27, pp. 46-99, 2007.

[7] P. Rapisarda and J. C. Willems, "State maps for linear systems," SIAM Journal on Control Optim., vol. 35, no. 3, pp. 1053 - 1091, 1997.

[8] T. H. Hughes, "Behavioral realizations using companion matrices and the Smith form," Accepted, SIAM Journal on Control Optim., 2016.

[9] J. C. Willems, "Dissipative dynamical systems, Part II: Linear systems with quadratic supply rates," Arch. Ration. Mech. Anal., vol. 45, pp. $352-393,1972$.

[10] D. C. Youla, L. J. Castriota, and H. J. Carlin, "Bounded real scattering matrices and the foundations of linear passive network theory," Trans. IRE Circuit Theory, vol. 4, pp. 102 - 124, 1959.

[11] B. D. O. Anderson and S. Vongpanitlerd, Network Analysis and Synthesis. Upper Saddle River, NJ: Prentice-Hall, 1973.

[12] J. C. Willems, "Least squares stationary optimal control and the algebraic Riccati equation," IEEE Trans. on Automatic Control, vol. 16, pp. 621 - 634, December 1971 (Special issue on the Linear-QuadraticGaussian Problem).

[13] _ - "On the existence of a nonpositive solution to the Riccati equation," IEEE Trans. on Automatic Control, vol. 19, pp. 592 - 593, 1974.

[14] J. Collado, R. Lozano, and R. Johansson, "On Kalman-YakubovichPopov lemma for stabilizable systems," IEEE Trans. on Automatic Control, vol. 46, no. 7, pp. 1089 - 1093, July 2001.

[15] L. Pandolfi, "An observation on the positive real lemma," Journal of Mathematical Analysis and Applications, vol. 255, no. 2, pp. 480-490, Mar 2001.

[16] S. Kunimatsu, K. Sang-Hoon, T. Fujii, and M. Ishitobi, "On positive real lemma for non-minimal realization systems," Proceedings of the 17th IFAC World Congress, Seoul, pp. 5868 - 5873, 2008.

[17] W. Sun, P. P. Khargonekar, and D. Shim, "Solution of the positive real control problem for linear time-invariant systems," IEEE Trans. on Automatic Control, vol. 39, no. 10, pp. 2034 - 2046, October 1994.

[18] A. Ferrante, "Positive real lemma: Necessary and sufficient conditions for the existence of solutions under virtually no assumptions," IEEE Trans. on Automatic Control, vol. 50, pp. 720 - 724, May 2005.

[19] A. Ferrante and L. Pandolfi, "On the solvability of the positive real lemma equations," Systems and Control Letters, vol. 47, no. 3, pp. 211 - 219, October 2002.

[20] S. Karikalan, M. N. Belur, C. D. Athalye, and R. A. Razak, "Uncontrollable dissipative systems: observability and embeddability," International Journal of Control, vol. 87, no. 1, pp. 101 - 119, 2014.

[21] D. Pal and M. N. Belur, "Dissipativity of uncontrollable systems, storage functions, and Lyapunov functions," SIAM Journal on Control Optim., vol. 47, no. 6, pp. $2930-2966,2008$.

[22] J. C. Willems and H. L. Trentelman, "On quadratic differential forms," SIAM Journal on Control Optim., vol. 36, no. 5, pp. 1703 - 1749, September 1998.

[23] A. J. van der Schaft and P. Rapisarda, "State maps from integration by parts," SIAM Journal on Control Optim., vol. 49, no. 6, pp. 2415 - 2439, 2011. 